\title{
Editorial
}

\section{Maintaining Professional Identity \& Integrity within the Field of Health Promotion}

Health promotion, or the process of helping healthy people stay healthy, is a growing and exciting field. Despite the budget crisis in California, the job outlook for health promotion professionals continues to improve with the increased variety of settings in which health promotion is integrated (i.e. clinics, hospitals, corporations, school, communities). Also, health promotion programs, especially those in county health departments, are heavily funded and supported by the federal government. With more and more health-related professionals joining forces with us, it's important that we understand just what sets health education and health promotion apart from other health-related fields such as exercise science, physical education, allied health, nutrition, and so forth. After quite a bit of professional soul searching, it appears to me that there are three predominant and fundamental characteristics that make health promotion unique and largely sets it apart from other health-related fields. Health promotion is heavily prevention-based (i.e., educational and modification efforts), process-oriented (i.e., program planning), and has a multidimensional and therefore multidisciplinary (i.e., holistic) nature. The acceptance of these characteristics by our colleagues in other health-related fields is imperative and perpetuates the "joining of forces" among a variety of professionals.

Collaborating and joining forces provides for stronger efforts and more effective outcomes with regards to the status of our nation's health. However, it is understandable that when health promotion and health education is introduced into or joined with a separate but related field, there may be initial resistance due to a lack of understanding. Health educators within physical education, exercise science, medical or other health-related settings have experienced struggles to maintain their professional identity as well as the integrity of the field. Although I may be "preaching to the choir," accepting differences, especially pertaining to philosophy, in our respective professions is absolutely essential to the continued growth and development of our field. Recognizing, accepting, respecting, and valuing differences results in strengthened relationships between professions, which ultimately improves the overall effectiveness of our respective efforts. All of us in the health care field need to recognize and value differences in our educational preparation, professional experience, research interests and expectations. Although our colleagues in nutrition, exercise science, psychology, physical education and so forth have an obvious and substantial interest in health, the background of health promotion professionals may provide for a much different philosophy, one that reflects the three characteristics described above.

Maintaining our professional identity and integrity requires that our efforts truly reflect the basic concepts of health promotion, despite possible misunderstandings from our colleagues in other health-related fields, and is fundamental to the continued progression and credibility of the health promotion field.

Kristine Brown

CJHP Associate Editor 